\title{
BMJ Open Stress, anxiety and depression among healthcare workers facing COVID-19 pandemic in Egypt: a cross-sectional online-based study
}

\author{
Hebatalla Mohamed Aly (D) , ${ }^{1}$ Nader Attia Nemr, ${ }^{2}$ Rania Mohammed Kishk, ${ }^{3}$ \\ Noha Mohamed Abu bakr Elsaid ${ }^{1}$
}

To cite: Aly HM, Nemr NA, Kishk RM, et al. Stress, anxiety and depression among healthcare workers facing COVID-19 pandemic in Egypt: a cross-sectional online-based study. BMJ Open 2021;11:e045281. doi:10.1136/ bmjopen-2020-045281

- Prepublication history and additional supplemental material for this paper are available online. To view these files, please visit the journal online (http://dx.doi.org/10.1136/ bmjopen-2020-045281).

Received 02 October 2020 Revised 10 March 2021 Accepted 22 March 2021

Check for updates

(C) Author(s) (or their employer(s)) 2021. Re-use permitted under CC BY-NC. No commercial re-use. See rights and permissions. Published by BMJ.

${ }^{1}$ Public Health, Community, Occupational and Environmental Medicine, Suez Canal University Faculty of Medicine, Ismailia,

Egypt

${ }^{2}$ Endemic and Infectious Diseases, Suez Canal University Faculty of Medicine, Ismailia, Egypt

${ }^{3}$ Microbiology and Immunology, Suez Canal University Faculty of Medicine, Ismailia, Egypt

Correspondence to Dr Hebatalla Mohamed Aly; dr.hebatalla@gmail.com

\section{ABSTRACT}

Objective This study assessed perceived stress, anxiety and depression among healthcare workers facing the COVID-19 pandemic in Egypt.

Setting This was an online study where a Google form was prepared including sociodemographic and occupational data as well as three validated questionnaires to assess perceived stress, anxiety and depression, respectively. The form was distributed online to all social media groups including healthcare workers all across the country, and responses were collected until the sample size of 262.

Participants Healthcare workers (physicians, dentists, pharmacists, physiotherapists, nurses, technicians and administrators) working in governmental or educational hospitals from all Egyptian governorates who are members of social media groups. The mean age of participants was $33.4 \pm 5.9$ years, $70 \%$ were women, about $70 \%$ were married and $66 \%$ were physicians.

Outcomes The frequency of perceived stress, anxiety and depression observed among the study participants according to the results of their questionnaires. Then the frequencies were compared between different sociodemographic characteristics.

Results Only $1.3 \%$ showed low perceived stress while $98.5 \%$ showed moderate to severe stress. About $9.5 \%$ did not experience generalised anxiety, while the remaining $90.5 \%$ had different degrees of anxiety as mild anxiety showed the highest per cent affecting about $40 \%$ of participants followed by moderate anxiety about $32 \%$ then severe anxiety, $18.5 \%$. With regard to depression, $94 \%$ of participants showed mild to severe depression.

Conclusion This study showed a high prevalence of perceived stress, anxiety and depression among healthcare workers during the COVID-19 pandemic that affected all workers regardless of different sociodemographic characteristics.

\section{INTRODUCTION}

Coronaviruses are enveloped single-stranded RNA viruses of zoonotic nature that cause symptoms ranging from those similar to the common cold to more severe respiratory, enteric, hepatic and neurological symptoms. ${ }^{1}$ Other than SARS-CoV-2, there are six known
Strengths and limitations of this study

- The study included representatives from all occupational categories of healthcare workers (physicians, dentists, pharmacists, nurses, technicians, chemists and administrators).

- The study also included workers from almost all Egyptian governorates.

- To our knowledge, this is one of the early papers assessing the actual frequency of stress, anxiety and depression among healthcare workers during the peak of the pandemic in Egypt.

- Representation of the different occupational categories was not equal, as the study sample included $66 \%$ physicians and the remaining included different healthcare staff members. Therefore, it was not possible to make a comparison between the different occupational categories regarding mental disorders.

- The study was performed through an online form, and thus some data may be lost due to healthcare members who may have been offline during this period or may not have been interested in social media use.

coronaviruses known to humans: $\mathrm{HCoV}-$ 229E, HCoV-OC43, SARS-CoV, HCoVNL63, HCoV-HKU1 and MERS-CoV. ${ }^{2}$ Coronavirus has caused two large-scale pandemics in the past two decades: SARS and MERS. ${ }^{3}$ In December 2019, a cluster of patients with idiopathic pneumonia was reported. They were linked to the South China local Huanan seafood market in Wuhan, Hubei Province, China. ${ }^{1}$

The WHO announced the occurrence of the novel coronavirus and declared it a Public Health Emergency of International Concern under the International Health Regulation on 30 January 2020. The novel coronavirus was officially named by the WHO as COVID-19 on 11 February 2020. The pandemic has not only caused a high mortality rate from viral 
infections but also psychological and mental effects on the rest of the world. ${ }^{4}$

On 30 May 2020, there were 5819962 confirmed cases of COVID-19 and 362786 confirmed deaths worldwide. In Egypt, there were 22082 confirmed cases of COVID-19 and 879 deaths in the same period. ${ }^{5}$ Egypt has implemented several preventative measures to fight COVID-19. The government of Egypt announced the extension of curfew across the country. It also announced the suspension of international air passenger arrivals. Progress has been made in expanding the number of peripheral laboratories able to test for COVID-19. Furthermore, Egypt maintained effective contact tracing with proper quarantine mechanisms, based on the current well-structured Infection Prevention and Control programme and continued systematic testing of patients seeking treatment for all acute respiratory infections. ${ }^{6}$

The pandemic had resulted in the prevalence of a wide range of psychological problems such as fear, anxiety, stigma, prejudice, marginalisation towards the disease, and its relation to all people from healthy individuals and at-risk individuals to care workers. Mass quarantine could cause a sense of mass hysteria, fear and anxiety in healthcare workers (HCWs) working in hospitals as well as isolation units. ${ }^{7}$ Medical HCWs who are exposed and have direct contact with confirmed and suspected coronavirus cases are called frontline HCWs. They are prone to increased workload, higher risk of infection and mental health problems. ${ }^{8}$

The COVID-19 pandemic has resulted in unprecedented psychological stress on HCWs, such as anxiety, fear, panic attacks, post-traumatic stress symptoms, psychological distress, stigma, avoidance of contact, depressive tendencies, sleep disturbances, helplessness, interpersonal and isolation from family and social support, as well as concerns about their friends and family being exposed to infection. ${ }^{9}$ Although mental health problems and psychosocial issues are common among HCWs, most health professionals do not often seek or receive regular mental healthcare. ${ }^{10}$

In addition, the mental health problems of HCWs would negatively affect their attention, cognitive functioning and clinical decision-making, leading to a subsequent increase in the incidence of medical errors and incidents, and thus putting patients at risk. ${ }^{11}{ }^{12}$ It was also well known that acute stress in disasters can have a longterm effect on overall well-being. ${ }^{13}$ Therefore, the mental health problems of HCWs in the COVID-19 pandemic have become an urgent public health concern.

Previous studies reported adverse psychological reactions to the 2003 SARS outbreak among HCWs. ${ }^{14}$ Studies showed that these HCWs were afraid of contagion and infecting their family, friends and colleagues, felt uncertainty and stigmatisation, reported reluctance to work or contemplating resignation. In addition, they reported increased high levels of stress, anxiety and depression symptoms, which could have long-term psychological implications. ${ }^{13} 15$ Psychological assistance services, including telephone, internet and application-based intervention, have been widely deployed by local and national mental health institutions in response to the COVID-19 outbreak. On 2 February 2020, the State Council of China has announced the establishment of nationwide psychological assistance hotlines to help during the pandemic. However, mental health interventions targeting HCWs are relatively scarce. ${ }^{16}$

That is why addressing mental health issues of HCWs is essential to improving pandemic prevention as well as control. ${ }^{17}$ To date, research on the psychological impact of COVID-19 on HCWs is still under investigation. The current study aims to evaluate mental health outcomes among HCWs who interact with patients with COVID-19 by quantifying the magnitude of symptoms of depression, anxiety, and distress and by analysing the potential risk factors associated with these symptoms.

\section{Study objectives}

1. To assess perceived stress among HCWs facing the COVID-19 pandemic in governmental or educational hospitals.

2. To assess general anxiety among HCWs facing the COVID-19 pandemic in governmental or educational hospitals.

3. To assess depression among HCWs facing the COVID-19 pandemic in governmental or educational hospitals.

4. To find out the sociodemographic and occupational risk factors for stress, anxiety, as well as depression, among HCWs facing the COVID-19 pandemic in governmental or educational hospitals.

\section{MATERIALS AND METHODS \\ Study design}

A web-based cross-sectional survey to assess perceived stress, general anxiety and depression among HCWs facing the COVID-19 pandemic in Egypt.

\section{Study setting}

All governmental and educational hospitals dealing with suspected or confirmed cases of COVID-19 or their contacts in Egypt.

\section{Study population}

HCWs (physicians, pharmacists, dentists, physiotherapists, nurses, technicians, chemists and administrators) in the previously mentioned hospitals in Egypt dealing with suspected and confirmed cases of COVID-19 or their contacts.

\section{Sampling}

Sample size was calculated using Epi Info V.7 using the prevalence of anxiety, depression and perceived stress (20.2, $12.7 \%$ and $59 \%$, respectively), ${ }^{18}$ with a confidence level $90 \%$ and a margin of error of $5 \%$, and the study population is assumed to be 330000 (which represents about 82000 physicians and 250000 nurses working in 
the Ministry of Health, which has the greatest per cent of HCWs). The highest sample size obtained was 262 participants.

\section{Sampling technique}

The link to the form was distributed to all social media and email groups including HCWs starting from May 2020. Responses were collected until the completion of the required sample on 22 June 2020. Three hundred and sixteen participants were collected.

\section{Patient and public involvement}

Participants were not involved in the design, conduct or documentation of the study. The title as well as the objectives of the paper was announced on social media groups including the target population, and the link was added.

The Google form link included informed consent about the study and the authors at the beginning, and participants have to agree to consent before proceeding with the questionnaires. Three hundred and sixteen complete responses were received representing all healthcare categories that deal with patients or their contacts during the
COVID-19 pandemic in Egypt's governmental or educational hospitals.

The mean age of the study participants was $33.4 \pm 5.9$ years, $70 \%$ were women and about $70 \%$ were married (table 1). Participants represented HCWs from all over Egypt, as all governorates were represented with varying frequencies, with Ismailia showing the most frequent representation. The distribution of occupational categories for participating HCWs was as follows: physicians (66 $\%$ ) followed by other medical staff (pharmacists, dentists and physiotherapists) representing approximately $18 \%$ of the sample. The representation of nurses, technicians, chemists and hospital administration staff was lower (15.5\% collectively). Among the physicians, paediatrics, chest, and anaesthesia and intensive care unit were the most frequent specialties ranging from $6 \%$ to $11 \%$ of the participating physicians.

After completing the study, the results and recommendations were published on the same social media groups that included the participants.

\section{Methods}

Four questionnaires were used:

Table 1 Sociodemographic characteristics of participants $(n=316)$

\begin{tabular}{|lll}
\hline Characteristic & Frequency & Per cent \\
\hline $\begin{array}{l}\text { Age in years } \\
<30\end{array}$ & 75 & 23.7 \\
\hline 30 & 241 & 69.9 \\
\hline Mean \pm SD & $33.4 \pm 5.9$ & \\
\hline Gender & & 29.7 \\
\hline Male & 94 & 70.3 \\
\hline Female & 222 & 24.1 \\
\hline Marital status & & 69.9 \\
\hline Single & 76 & 6 \\
\hline Married & 221 & 10.8 \\
\hline Divorced/widow & 19 & 89.2 \\
\hline Having offspring (n=240) & & 23.8 \\
\hline No & 26 & 47.7 \\
\hline Yes & 214 & 22 \\
\hline Number of offspring (214) & & 6.5 \\
\hline 1 & 51 & 66.1 \\
\hline 2 & 102 & 18.4 \\
\hline 3 & 47 & 6 \\
\hline 4 & 14 & 4.4 \\
\hline Occupational category & & 5.1 \\
\hline Physicians & 209 & \\
\hline Other medical staff (dentists, pharmacists, physiotherapists) & 58 & 19 \\
\hline Nurses & 14 & 16 \\
\hline Technicians & & \\
\hline Administrative & & \\
\hline
\end{tabular}




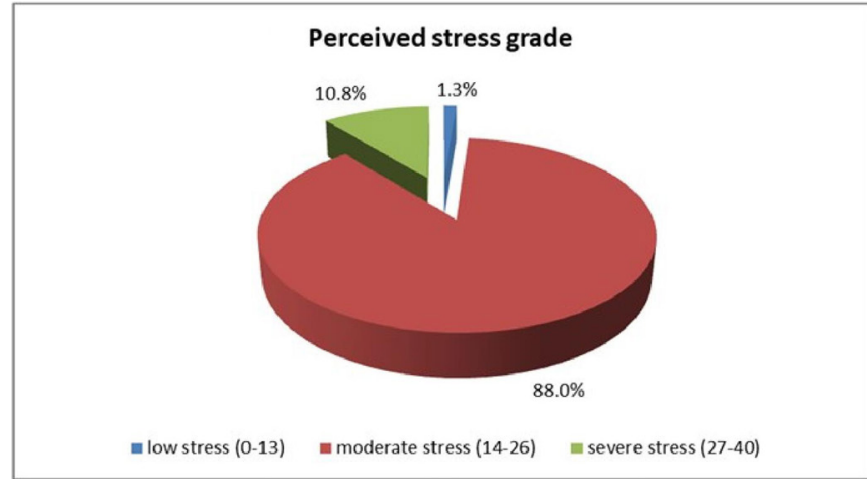

Figure 1 Perceived stress grade among study participants $(n=316)$.

1. Sociodemographic questionnaire (age, gender, marital status, occupational data like specialisation as well as place of work to know the representative governorate).

2. Perceived Stress Scale (PSS-10): consists of 10 questions about feelings and thoughts during the last month. Each case was asked to indicate how often they felt or thought in a certain way. The answers to each question are on a 5-rate scale, ranging from never to very often. Each answer has a score then the total score is calculated. Scores ranging from 0 to 13 are considered low stress. Scores 14-26 are considered moderate stress. Scores between 27 and 40 are considered highperceived stress. ${ }^{19}$ We used a validated Arabic version. ${ }^{20}$

3. Generalized Anxiety Disorder 7-item questionnaire (GAD-7): it consists of seven questions on feeling in the last 2 weeks. Answers are on a 4-rate scale ranging from not at all sure to nearly every day. Each answer has a score then a total score will be calculated. ${ }^{82122} \mathrm{We}$ used a validated version in the Arabic. ${ }^{23}$

4. Patient Health Questionnaire (depression module) 9 (PHQ-9): it consists of nine questions about feeling in the past 2 weeks. Answers are on a 4-rate scale, ranging from not at all to nearly every day. Each answer has a score then the total score is calculated. A score of 1-4 indicates minimal depression. A score of 5-9 indicates mild depression. A score of 10-14 indicates moderate depression. A score of 15-19 indicates moderately severe depression. A score of 20-27 indicates severe depression. ${ }^{24}$ We used a validated version in Arabic. ${ }^{23}$

- All four questionnaires were displayed in one link on the Google form, including informed consent, and published among HCWs (online supplemental material 1). The form link is: (https://docs.google.com/ forms/d/e/1FAIpQLSebBnVavcJmdu00v0TtZ6rGOF os5w1ph6aIBFsYDRxIfX-DiQ/viewform?usp=sf_link).

- Reminders were sent to participants to fill out the form, either through group posts or the inbox of members who were randomly selected.

- To avoid losing data, the form was designed so that the answer to a question is mandatory to proceed to the next one, except for optional questions such as the name. Consequently, all 316 responses were complete with no data loss.
Table 2 Stress score according to Perceived Stress Scale (PSS-10), anxiety score according to Generalized Anxiety Disorder 7-item questionnaire (GAD-7) and depression score according to Patient Health Questionnaire 9 (PHQ-9)

\begin{tabular}{lll}
\hline Score & Mean \pm SD & Median (range) \\
\hline PSS-10 score & $22.1 \pm 3.9$ & $22(0-33)$ \\
GAD-7 score & $10.3 \pm 4.7$ & $10(1-21)$ \\
PHQ-9 score & $12.6 \pm 5.8$ & $12(0-27)$ \\
\hline
\end{tabular}

- We have activated the 'limit to one response' option to avoid duplicate responses.

\section{Data management}

All data were entered in SPSS V.23. Quantitative data, such as age, were presented as mean and SD; while qualitative data, such as the frequency of perceived stress or depression, were presented as frequency and percentage. The difference between the frequency of mental health disorders among different sociodemographic categories was assessed by the $\mathrm{X}^{2}$ test. Spearman's correlation coefficient was used to study the strength of the association between different scores of mental disorders studied.

\section{Ethical considerations}

All the following ethical considerations were considered:

- Informed consent was written at the beginning of the Google form and participants chose if they agree or disagree to fill the form.

- Confidentiality of the collected data: the collected data were kept secret for research use only.

- The participants were informed that responding is voluntary and that they can refuse to respond without stating any reason.

- The aims of the research were achieved without disturbing the harmony of the work rhythm.

- Feedback about the results of the study was announced to groups of participants at the end of the study.

\section{RESULTS}

Stress, anxiety and depression assessed among the participants using validated questionnaires, which are PSS-10, GAD-7 and PHQ-9, respectively, as well as the scores, are shown in table 2.

The grades of stress, depression and anxiety are shown in figures 1-3. As shown in figure 1, only $1.3 \%$ show low perceived stress, while $98.5 \%$ show moderate to severe stress. Figure 2 shows that $9.5 \%$ have no generalised anxiety, while the remaining $90.5 \%$ have different degrees of anxiety, whereas mild anxiety showed the highest percentage affecting about $40 \%$ of participants followed by moderate anxiety about $32 \%$ then severe anxiety, $18.5 \%$. With respect to depression, about $6 \%$ of participants showed no or minimal degree of depression, and about $14 \%$ showed severe depression while the remaining $80 \%$ showed varying grades, from mild to moderately severe. 
Generalized anxiety grade

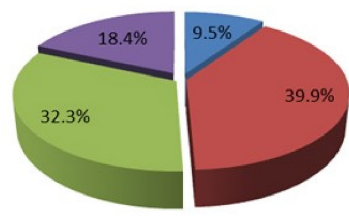

$\|$ No anxiety $(<5) \|$ mild anxiety $(5-9) \|$ moderate anxiety $(10-14) \|$ severe anxiety $(\geq 15)$

Figure 2 Generalized Anxiety Disorder grade among study participants $(n=316)$.

The coincidence of mental health disorders among study participants was assessed. The cut-off values used were: perceived stress score $(\geq 14)$, anxiety score $(\geq 5)$ and depression score $(\geq 5)$. It was found that about $87 \%$ suffer from all the three disorders (stress, anxiety and some degree of depression), and only 3.5\% suffer from one disorder.

The same cut-off values were used to compare the presence of each mental health problem between the different sociodemographic characteristics (table 3). The difference in the frequency of mental health problems between the different sociodemographic characteristics did not show a statistically significant difference.

\section{DISCUSSION}

This cross-sectional online survey received 316 responses. It was designed to investigate the impact of COVID-19 on mental health among HCWs during the COVID-19 pandemic. To our knowledge, this study is one of the leading studies to discuss this issue in Egypt. The study was conducted in the middle of the first wave of the pandemic in Egypt in the period from the beginning of May 2020 to the end of June 2020.

This study found a significant prevalence of the studied mental health problems of perceived stress, anxiety and depression: $98.5 \%, 90.5 \%$ and $94 \%$, respectively. These findings were consistent with another study conducted in Egypt just prior to our study in April, and it also revealed a high prevalence of mental health problems: stress,

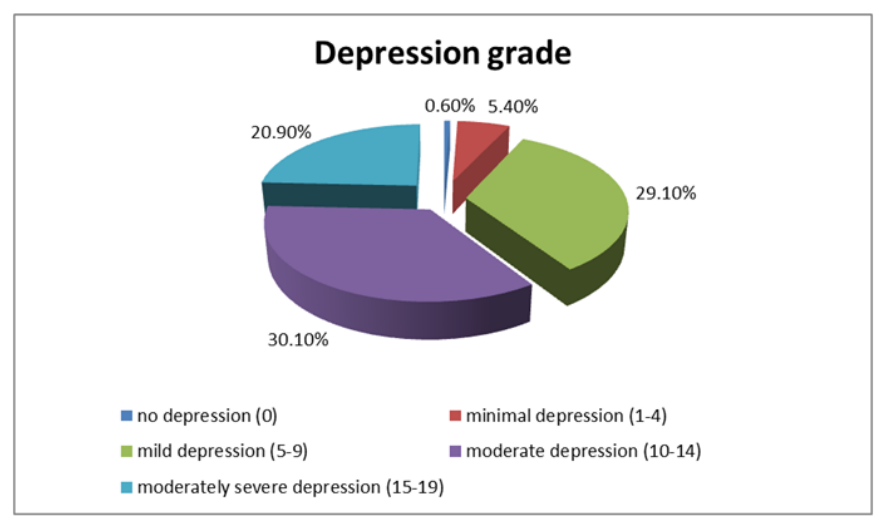

Figure 3 Depression grades among study participants $(n=316)$. anxiety and depression, 80.9\%, 76.4\%, 77.2\%, respectively, among HCWs. ${ }^{25}$ Therefore, the situation became more critical in Egypt as the number of confirmed and suspected cases increased later. Moreover, due to the shortage of HCWs in Egypt which was 0.5 per 1000 people for physicians and 1.9 per 1000 for nurses as reported by the World Bank, ${ }^{26}$ physicians from all specialties, even dentists and pharmacists, were transferred from their hospitals to participate in the diagnosis, treatment of suspected or confirmed case, and follow up of contacts of confirmed cases.

Previous studies in different countries also reported a higher prevalence of mental health problems among HCWs compared with the general population due to their close and frequent contact with patients, working longer hours than usual, and continuous working in frightening, stressful and constrained conditions where they are exposed to constant threat of infection. 892728

Some studies showed a high prevalence of mental health problems among HCWs, nonetheless, the frequencies found in the current study were much higher than other worldwide studies. For example, a previous study conducted in Italy investigated mental health symptoms during COVID-19 among HCWs who reported a prevalence of stress $(21.90 \%)$, anxiety $(19.80 \%)$ and depression $(24.73 \%) .{ }^{29}$ Another systematic review conducted by Luo et al in $2020^{30}$ found that the combined prevalence of anxiety and depression was 33\% (95\% CI: $28 \%$ to $38 \%$ ) and $28 \%$ (95\% CI: $23 \%$ to $32 \%$ ), respectively. However, the prevalence of anxiety and depression in this review was similar between HCWs and the general public, which contradicts the previously mentioned studies of HCWs. ${ }^{30}$

Another study of physicians and nurses in Wuhan reported a high prevalence of stress $(71.5 \%)$, anxiety $(44.6 \%)$ and depression $(50.4 \%)^{28}$; nevertheless, this is still lower than the prevalence detected in our study and this can be attributed to the reason that $70.3 \%$ of our participants were women, $69.9 \%$ of them were married with $89.2 \%$ had offspring. All these characteristics were considered risk factors for higher psychological impact in a recent Egyptian study. ${ }^{31}$ The mean age of the current study participants was $33.4 \pm 5.9$ years which indicates that participants had junior titles, and most of them had fewer years of work experience; this was consistent with Mazza et al who state that young age has more tendency to be stimulated by surrounding stressors. ${ }^{32}$

Other risk factors for increasing mental health problems among HCWs in Egypt include an insufficient number of HCWs in Egypt leading to an increased workload and insufficient personal protective equipment (PPE) leading to an increased fear of infection risk. ${ }^{33}$ An Egyptian study that assessed the knowledge, attitude and perception of HCWs during COVID-19 found that despite the high degree of knowledge of physicians, the vast majority of HCWs were frightened and felt more likely to have COVID-19 infection (83.1\% and $89.2 \%$, respectively). Additionally, it showed that the unavailability of PPE, fear of transmitting disease to their families, social stigma, and 
Table 3 Stress, anxiety and depression among different sociodemographic characteristics

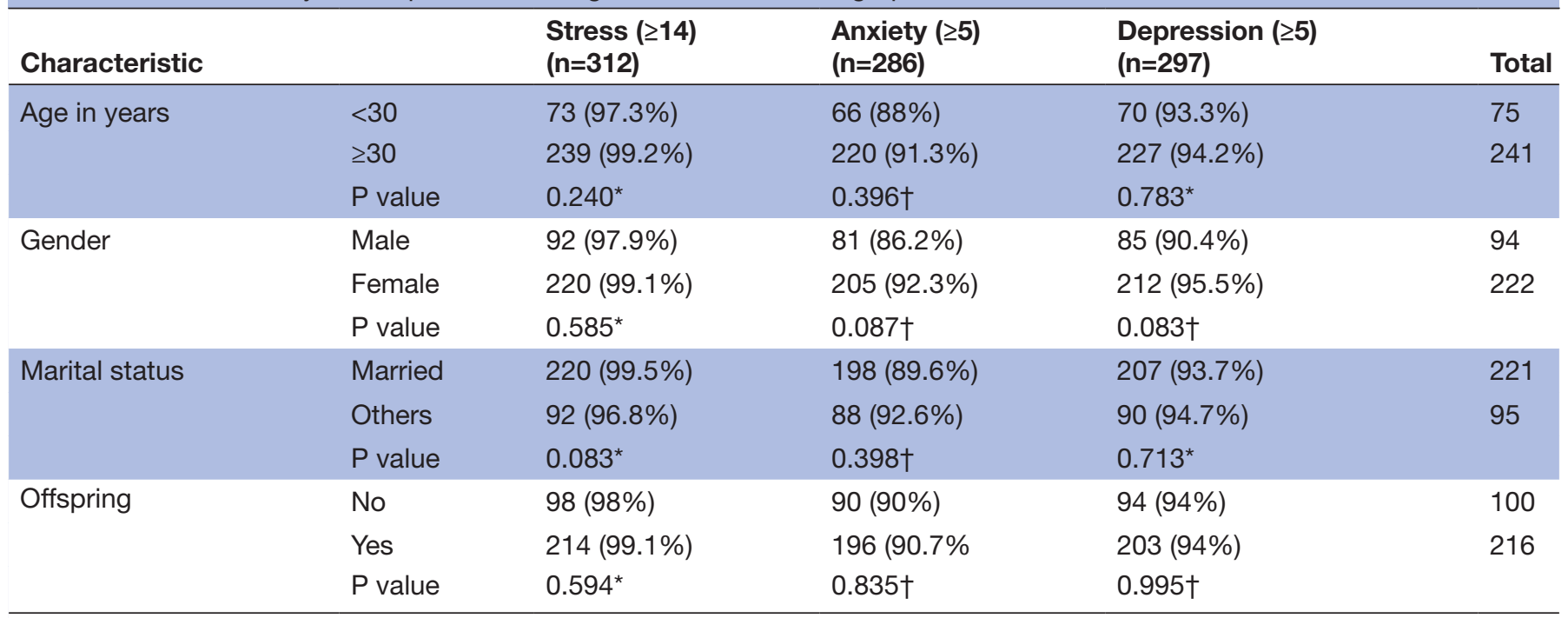

${ }^{*}$ Fisher's exact test.

$\dagger \mathrm{X}^{2}$ test.

unsuitable workplace circumstances such as crowdedness and poor ventilation were the most commonly reported reasons for increased risk perception. ${ }^{34}$

Finally, the increased prevalence of mental health problems among study participants may also be related to the study period which extends from the beginning of May to the end of June where the situation in Egypt has become more critical with the increasing number of cases and deaths despite the probability of lower reported cases than the actual numbers. ${ }^{35}$ In addition, the number of doctors with confirmed infection during this period was reported to be about 430 with 68 deaths, with a mortality rate of $15.8 \%$ according to the Egyptian Medical Syndicate report, and this is considered high. This may also illustrate the higher level of stress, anxiety and depression among the HCWs in our study. ${ }^{36}$

Although the Egyptian Ministry of Health announced on 31 March that two hotlines for mental health were allocated in the General Secretariat to provide psychological support to all citizens including HCWs during the COVID-19 pandemic, ${ }^{25} 37$ our results showed that this was insufficient and comprehensive interventions are still needed.

The difference in the frequency of perceived stress, anxiety and depression among the study participants was compared with regard to different sociodemographic characteristics as age and gender but the difference was not statistically significant. This may indicate the impact of the pandemic on the mental health of all HCWs during this period regardless of different sociodemographic characteristics.

\section{Limitations of the study}

Representation of the different occupational categories was not equal as the study sample included $66 \%$ of physicians and the rest included different healthcare staff members, and thus it was not possible to make a comparison between the different occupational categories regarding mental health problems.

The study was performed via an online form and this was missed by some healthcare member participants, who may have been offline during this period or who may not have been interested in using social media.

\section{CONCLUSION}

This study showed the high prevalence of perceived stress, anxiety and depression among HCWs during the COVID-19 pandemic that affected all workers regardless of different sociodemographic characteristics.

\section{RECOMMENDATIONS}

- Targeted interventions are needed to enhance the psychological health of HCWs and reinforce the capacity of healthcare system during the pandemic.

- Adequate support should be ensured, appropriate education and training provided, in addition to guaranteeing adequate resources.

- Moreover, psychosocial needs should be monitored, and psychosocial services can be delivered via telemedicine.

Contributors HMA-writing the protocol, ethics committee approval, data collection, data analysis and results writing, paper writing and editing. NANshared in data collection. RMK-shared in protocol writing and data collection. NMAbE-shared in writing protocol, ethics committee approval, data collection, and paper writing and editing.

Funding The authors have not declared a specific grant for this research from any funding agency in the public, commercial or not-for-profit sectors.

Competing interests None declared.

Patient consent for publication Not required. 
Ethics approval The study was approved by the Ethics Committee of the Faculty of Medicine, Suez Canal University on 5 May 2020 with approval number 4168.

Provenance and peer review Not commissioned; externally peer reviewed.

Data availability statement Data are available upon reasonable request. All participants' data are coded and are available in an Excel sheet. The ethics committee-approved protocol is available and data analysis output sheet is available. All these data are already present with the corresponding author; if there is reasonable request, data will be sent.

Supplemental material This content has been supplied by the author(s). It has not been vetted by BMJ Publishing Group Limited (BMJ) and may not have been peer-reviewed. Any opinions or recommendations discussed are solely those of the author(s) and are not endorsed by BMJ. BMJ disclaims all liability and responsibility arising from any reliance placed on the content. Where the content includes any translated material, BMJ does not warrant the accuracy and reliability of the translations (including but not limited to local regulations, clinical guidelines, terminology, drug names and drug dosages), and is not responsible for any error and/or omissions arising from translation and adaptation or otherwise.

Open access This is an open access article distributed in accordance with the Creative Commons Attribution Non Commercial (CC BY-NC 4.0) license, which permits others to distribute, remix, adapt, build upon this work non-commercially, and license their derivative works on different terms, provided the original work is properly cited, appropriate credit is given, any changes made indicated, and the use is non-commercial. See: http://creativecommons.org/licenses/by-nc/4.0/.

\section{ORCID iD}

Hebatalla Mohamed Aly http://orcid.org/0000-0003-3949-6480

\section{REFERENCES}

1 Zhu N, Zhang D, Wang W, et al. A novel coronavirus from patients with pneumonia in China, 2019. N Engl J Med Overseas Ed 2020;382:727-33.

2 Li Q, Guan X, Wu P, et al. Early transmission dynamics in Wuhan, China, of novel Coronavirus-Infected pneumonia. N Engl J Med 2020;382:1199-207.

3 Zhou P, Yang X-L, Wang X-G, et al. A pneumonia outbreak associated with a new coronavirus of probable bat origin. Nature 2020;579:270-3.

4 Xiao C. A novel approach of consultation on 2019 novel coronavirus (COVID-19)-related psychological and mental problems: structured letter therapy. Psychiatry Investig 2020;17:175-6.

5 World Health Organization. Coronavirusdisease 2019 (COVID-19) situation report 131. World Health Organization, 2020b. Available: https://www.who.int/docs/default-source/coronaviruse/situationreports/20200530-covid-19-sitrep-131.pdf?sfvrsn=d31ba4b3_2

6 WHO, EMRO. Who delegation concludes COVID-19 technical mission to Egypt, 2020. Available: http://www.emro.who.int/media/ news/who-delegation-concludes-covid-19-technical-mission-toegypt.html

7 Mak IWC, Chu CM, Pan PC, et al. Long-Term psychiatric morbidities among SARS survivors. Gen Hosp Psychiatry 2009;31:318-26.

8 Wang D, Hu B, Hu C, et al. Clinical characteristics of 138 hospitalized patients with 2019 novel coronavirus-infected pneumonia in Wuhan, China. JAMA 2020;323:1061-9.

9 Zheng W. Mental health and a novel coronavirus (2019-nCoV) in China. J Affect Disord 2020;269:201-2.

10 Xiang Y-T, Yang Y, Li W, et al. Timely mental health care for the 2019 novel coronavirus outbreak is urgently needed. The Lancet Psychiatry 2020;7:228-9.

11 Panagioti M, Geraghty K, Johnson J, et al. Association between physician burnout and patient safety, professionalism, and patient satisfaction: a systematic review and meta-analysis. JAMA internal medicine 2018;178:1317-31.

12 LeBlanc VR. The effects of acute stress on performance: implications for health professions education. Academic Medicine 2009;84:S25-33.

13 Mulfinger N, Sander A, Stuber F, et al. Cluster-Randomised trial evaluating a complex intervention to improve mental health and wellbeing of employees working in hospital - a protocol for the SEEGEN trial. BMC Public Health 2019;19:1694.
14 Lee AM, Wong JGWS, McAlonan GM, et al. Stress and psychological distress among SARS survivors 1 year after the outbreak. Can $J$ Psychiatry 2007;52:233-40.

15 Maunder R, Hunter J, Vincent L, et al. The immediate psychological and occupational impact of the 2003 SARS outbreak in a teaching hospital. Cmaj 2003;168:1245-51.

16 The State Council of China. A notification to set up nationwide psychological assistance hotlines against the 2019-nCoV outbreak, 2020. Available: http://www.gov.cn/xinwen/2020-02/02/content 5473937.htm [Accessed April 2020].

17 Banerjee D. The COVID-19 outbreak: crucial role the psychiatrists can play. Asian J Psychiatr 2020;50:102014.

18 Du J, Dong L, Wang T, et al. Psychological symptoms among frontline healthcare workers during COVID-19 outbreak in Wuhan. Gen Hosp Psychiatry 2020;67:144-5.

19 Cohen S, Williamson G. Perceived stress in a probability sample of the United States. In: Spacapan S, Oskamp S, eds. The social psychology of health. Newbury Park, CA: SAGE, 1988.

20 Chaaya M, Osman $\mathrm{H}$, Naassan G, et al. Validation of the Arabic version of the Cohen perceived stress scale (PSS-10) among pregnant and postpartum women. BMC Psychiatry 2010;10:111.

21 Spitzer RL, Kroenke K, Williams JBW, et al. A brief measure for assessing generalized anxiety disorder. Arch Intern Med 2006;166:1092-7.

22 Wang HR, Cho H, Kim D-J. Prevalence and correlates of comorbid depression in a nonclinical online sample with DSM-5 Internet gaming disorder. J Affect Disord 2018;226:1-5

23 Sawaya $\mathrm{H}$, Atoui M, Hamadeh A, et al. Adaptation and initial validation of the patient health questionnaire - 9 (PHQ-9) and the generalized anxiety disorder - 7 questionnaire (GAD-7) in an Arabic speaking Lebanese psychiatric outpatient sample. Psychiatry Res 2016;239:245-52.

24 Kroenke K, Spintzer RL, Williams JB. The PHQ-9: validity of a brief depression severity measure. J Gen Intern Med 2001;16:606-13.

25 Elkholy H, Tawfik F, Ibrahim I, et al. Mental health of frontline healthcare workers exposed to COVID-19 in Egypt: a call for action. Int J of soc psychiatry. 2020: 20764020960192.

26 The World Bank. Open data | data, 2020. Available: https://data. worldbank.org/

27 Kumar A, Nayar KR. COVID 19 and its mental health consequences. $J$ Ment Health 2020;25:1-2.

28 Lai J, Ma S, Wang Y, et al. Factors associated with mental health outcomes among health care workers exposed to coronavirus disease 2019. JAMA Netw Open 2020;3:e203976.

29 Rossi R, Socci V, Pacitti F, et al. Mental health outcomes among frontline and second-line health care workers during the coronavirus disease 2019 (COVID-19) pandemic in Italy. JAMA Network Open 2020;3:e2010185.

30 Luo M, Guo L, Yu M, et al. The psychological and mental impact of coronavirus disease 2019 (COVID-19) on medical staff and general Public-A systematic review and meta-analysis. J Psychiatr Res 2020;7:113190.

31 El-Zoghby SM, Soltan EM, Salama HM. Impact of the COVID-19 pandemic on mental health and social support among adult Egyptians. J Community Health 2020;28:31.

32 Mazza C, Ricci E, Biondi S, et al. A nationwide survey of psychological distress among Italian people during the COVID-19 pandemic: immediate psychological responses and associated factors. Int J Environ Res Public Health 2020;17:3165.

33 Refeai SA, Kamal NN, Ghazawy ERA, et al. Perception and barriers regarding infection control measures among healthcare workers in Minia City, Egypt. Int J Prev Med 2020;11:11.

34 Abdel Wahed WY, Hefzy EM, Ahmed MI, et al. Assessment of knowledge, attitudes, and perception of health care workers regarding COVID-19, a cross-sectional study from Egypt. J Community Health 2020;45:1242-51.

35 Hassany M, Abdel-Razek W, Asem N, et al. Estimation of COVID-19 burden in Egypt. Lancet Infect Dis 2020;20:896-7.

36 Egyptian medical syndicate. Egyptian statistics, 2020. Available: https://www.care. gov.eg/EgyptCare/index.aspx [Accessed 2 may 2020].

37 Ahramonline. Egypt sets up hotlines for psychological support during coronavirus shutdown - Politics - Egypt, 2020. Available: http:// english. ahram.org.eg/NewsContent/1/64/366341/Egypt/Politics-/ Egypt-sets-up-hotlines-for-psychological-support-d.aspx 